\title{
Die APAF-Studie
}

\section{A Controlled Randomized Trial of Circumferential Pulmonary Vein Ablation Versus Antiarrhythmic Drug Therapy for Curing Paroxysmal Atrial Fibrillation: the Ablation for Paroxysmal Atrial Fibrillation (APAF) Trial}

Hintergrund und Problemstellung: Verschiedene Verfahren der Katheterablation sind in den letzten Jahren als kurative Therapieoption bei Patienten mit therapierefraktärem Vorhofflimmern (VHF) entwickelt worden. Die Wirksamkeit der antiarrhythmischen medikamentösen Therapien wurde in multiplen prospektiven Studien belegt. Zur Katheterab-

\begin{tabular}{|c|c|c|}
\hline \multicolumn{3}{|l|}{ Methodik } \\
\hline Studiendesign & \multicolumn{2}{|c|}{ Prospektive, randomisierte Single-Center-Studie } \\
\hline Primärer Endpunkt & \multicolumn{2}{|c|}{ Zeit bis zum Rezidiv von Vorhofflimmern } \\
\hline Patientenauswahl & \multicolumn{2}{|c|}{$\begin{array}{l}\text { Einschlusskriterien: paroxysmales } \\
\text { Vorhofflimmern für mindestens } 6 \text { Monate } \\
\text { Ausschlusskriterien: nicht benannt }\end{array}$} \\
\hline $\begin{array}{l}\text { Anzahl der teil- } \\
\text { nehmenden Zentren }\end{array}$ & \multicolumn{2}{|l|}{1} \\
\hline $\begin{array}{l}\text { Anzahl aller in die } \\
\text { Studie eingeschlossenen } \\
\text { Patienten }\end{array}$ & \multicolumn{2}{|l|}{150} \\
\hline $\begin{array}{l}\text { Ausgangsdaten: } \\
\text { Patienten- } \\
\text { charakteristika }\end{array}$ & \multicolumn{2}{|c|}{$\begin{array}{l}\text { Alter im Mittel } 55 \text { Jahre, Geschlecht und Dauer } \\
\text { des VHF gleich verteilt }\end{array}$} \\
\hline \multirow[t]{2}{*}{ LA Diameter } & Im Mittel $41 \mathrm{~mm}$ & \\
\hline & $\begin{array}{l}\text { Ablation } \\
\mathrm{n}=75\end{array}$ & $\begin{array}{l}\text { Medikation } \\
\mathrm{n}=75\end{array}$ \\
\hline $\begin{array}{l}\text { Spezifische Behandlung } \\
\text { im jeweiligen } \\
\text { Studienarm }\end{array}$ & $\begin{array}{l}\text { Zirkumferentielle } \\
\text { Pulmonalvenen- } \\
\text { ablation entweder } \\
\text { mit 8-mm- oder } \\
\text { "gekühlter" 4-mm- } \\
\text { Ablationselektrode }\end{array}$ & $\begin{array}{l}\text { Flecainid, Sotalol } \\
\text { oder Amiodaron }\end{array}$ \\
\hline
\end{tabular}

Ergebnisse $\left({ }^{*} p<0,05\right)$

\begin{tabular}{|c|c|c|}
\hline Follow-up ( 9 Monate) & Ablation & Medikation \\
\hline $\begin{array}{l}\text { Patientenzahl in den } \\
\text { randomisierten } \\
\text { Untergruppen }\end{array}$ & $\mathrm{n}=75$ & $\mathrm{n}=75$ \\
\hline $\begin{array}{l}\text { Anteil von Patienten } \\
\text { mit Sinusrhythmus } \\
\text { im Verlauf des klini- } \\
\text { schen Follow-up (täg- } \\
\text { liche EKG-Registrierung) } \\
\text { versus Patienten mit } \\
\text { VHF-Rezidiv }\end{array}$ & $\begin{array}{l}65 \text { vs. } 10 \\
\text { (8 Patienten mit VHF, } \\
2 \text { Patienten mit } \\
\text { permanenter } \\
\text { atrialer Tachy- } \\
\text { kardie) }\end{array}$ & 23 vs. 52 \\
\hline $\begin{array}{l}\text { Anteil der Patienten } \\
\text { mit Komplikationen }\end{array}$ & 1 TIA, 1 Perikarderguss & Nicht benannt \\
\hline
\end{tabular}

lation von Vorhofflimmern sind vor allem Single-Center-Erfahrungen publiziert. Die APAF-Studie sollte klären, ob die Katheterablation der medikamentösen Therapie hinsichtlich der Etablierung des Sinusrhythmus überlegen ist. Studienleiter war C. Pappone, Mailand, Italien.

\begin{tabular}{|c|c|c|}
\hline \multicolumn{3}{|c|}{ Evidence-based-Medicine- (EBM-) Score (www.TCTMD.com) } \\
\hline Klinischer primärer Endpunkt & $\mathrm{Ja}=3$, nein $=0$ & 3 \\
\hline Doppelblind (einschließlich Ärzten) & $\mathrm{Ja}=1$, nein $=0$ & 0 \\
\hline $\begin{array}{l}\text { Beobachtungsintervall für den } \\
\text { primären Endpunkt } \geq 6 \text { Monate }\end{array}$ & $\mathrm{Ja}=1$, nein $=0$ & 1 \\
\hline Multicenter (mindestens 3 Zentren) & $\mathrm{Ja}=1$, nein $=0$ & 0 \\
\hline $\begin{array}{l}\text { Externes und vom Steering } \\
\text { Committee unabhängiges Clinical } \\
\text { Event Committee / DSMB (Daten- } \\
\text { sicherheit Monitoring Board) }\end{array}$ & $\mathrm{Ja}=1$, nein $=0$ & 0 \\
\hline Primärer Endpunkt erreicht & $\mathrm{Ja}=1$, nein $=0$ & 1 \\
\hline $\begin{array}{l}\text { Power von } \geq 80 \% \text { für den } \\
\text { primären Endpunkt erreicht }\end{array}$ & $\mathrm{Ja}=1$, nein $=0$ & Unbekannt \\
\hline $\begin{array}{l}\text { Anteil des Follow-up der Patienten } \\
\text { für angiographischen primären } \\
\text { Endpunkt } \geq 80 \% \text { und } \geq 95 \% \text { für } \\
\text { klinischen primären Endpunkt }\end{array}$ & $\mathrm{Ja}=1$, nein $=0$ & 1 \\
\hline $\begin{array}{l}\text { Gesamt-EBM-Score } \\
\text { („Silber-Score“ für randomisierte Stuc }\end{array}$ & & 6 \\
\hline
\end{tabular}

Schlussfolgerung und Kommentar: Diese randomisierte Single-Centerstudie zeigte einen signifikanten Unterschied zwischen der primären Katheterablation und der antiarrhythmischen Medikation hinsichtlich des Erreichens von Sinusrhythmus innerhalb des Nachbeobachtungszeitraumes von 9 Monaten.

Die Bedeutung der primären, kurativ intendierten Katheterablation als Therapieoption wurde in dieser randomisierten Studie bei Patienten mit paroxysmalem Vorhofflimmern unterstrichen. Jedoch wurden wichtige Details des Studiendesigns und die Ergebnisse des Medikationsarmes in der Präsentation während der Late-Breaking-Trials-Session nicht vorgestellt, so dass eine abschließende Empfehlung zur Indikationsstellung weiterhin problematisch erscheint.

Herz 2006;31:166

DOI $10.1007 / \mathrm{s} 00059-006-2808-8$ 